\title{
Comparison of two exercise testing protocols in patients with chronic fatigue syndrome
}

\author{
Jo Nijs, PhD, PT; ${ }^{1-2 *}$ Kim Zwinnen, PT; ${ }^{1}$ Romain Meeusen, PhD; ${ }^{1}$ Bas de Geus, MSc; ${ }^{1}$ Kenny De Meirleir, PhD $^{1}$ \\ ${ }^{1}$ Department of Human Physiology, Faculty of Physical Education and Physiotherapy, Vrije Universiteit Brussel, Brussels, \\ Belgium; ${ }^{2}$ Division of Musculoskeletal Physiotherapy, Department of Health Care Sciences, University College Antwerp, \\ Antwerp, Belgium
}

\begin{abstract}
This study examined whether a linear exercise stress-testing protocol generated different peak exercise performance variables than a stepwise exercise testing protocol in patients with chronic fatigue syndrome (CFS). We conducted a comparative study with patients randomly allocated to one of two exercise testing protocols. Twenty-eight women with CFS completed two self-reported measures (the CFS Symptom List and the CFS Activities and Participation Questionnaire) and then performed until exhaustion either the linear or the stepwise exercise testing protocol with continuous monitoring of physiological variables (heart rate and oxygen uptake). At baseline, we found no significant differences in demographic features and health status between groups $(p>0.05)$. Based on ratio peak workload/peak oxygen uptake, mechanical efficiency was lower among the subjects performing the stepwise protocol $(p=0.002)$. When we analyzed the mean linear regression slope values between oxygen uptake and workload from each subject's minute-by-minute exercise data points, we found that mechanical efficiency was lower among the subjects performing the stepwise protocol $(p=0.039)$. Apart from mechanical efficiency, we found no differences in exercise performance data between groups $(p>0.05)$. Our results suggest that the difference between linear and stepwise exercise protocols cannot account for all discrepancies of previous studies on exercise performance data in women with CFS, but they do suggest that the nature of the exercise testing protocol influences mechanical efficiency in these patients. Further study is warranted.
\end{abstract}

Key words: assessment, cardiopulmonary exercise testing, chronic fatigue syndrome, exercise capacity, exercise performance, exercise physiology, fibromyalgia, measurement, mechanical efficacy, oxygen uptake.

\section{INTRODUCTION}

Chronic fatigue syndrome (CFS) is a debilitating disorder characterized by sleep impairments, fatigue, headache, muscle and joint aches, and symptoms of poor concentration and memory [1]. Symptoms are typically worsened after modest amounts of exercise [2], after increased daily physical activity [3], and after a maximal exercise stress test [4-5]. A delayed recovery from exercise typically occurs in patients with CFS [6].

Exercise performance testing is widely used for the assessment of patients with CFS and appears to be both reproducible and valid [7]. Exercise testing was able to distinguish between female patients with CFS and nondisabled sedentary females [8], and exercise capacity variables were correlated with activity limitations and participation restrictions in CFS patients [9]. Previous research has shown that patients with CFS present with an abnormal exercise

Abbreviations: $\mathrm{AMA}=$ American Medical Association, $\mathrm{CDCP}=$ Centers for Disease Control and Prevention, CFS = chronic fatigue syndrome, CFS-APQ = CFS Activities and Participation Questionnaire, HR = heart rate, ICC = intraclass correlation coefficient, K-S = Kolmogorov-Smirnov (goodness-of-fit test), RER = respiratory exchange ratio, $\mathrm{VCO}_{2}=$ rate of elimination of carbon dioxide, $\mathrm{VE}=$ ventilation, $\mathrm{VO}_{2}=$ oxygen uptake.

* Address all correspondence to Jo Nijs, PhD, PT; Vrije Universiteit Brussel, Mfys/Sport, KRO-gebouw -1, Laarbeeklaan 101, B-1090 Brussels, Belgium; +32-2-4774604; fax: +32-2-4774607. Email: Jo.Nijs@vub.ac.be

DOI: 10.1682/JRRD.2006.12.0153 
response: reduced peak oxygen uptake $\left(\mathrm{VO}_{2}\right)$ [8,10-11], reduction in peak heart rate (HR) [11], earlier exhaustion [11], reduced exercise tolerance [10,12], reduced workload [10], and accelerated glycolysis with increased lactate production [13]. Contrary to these findings, other studies show that the aerobic capacity of patients with CFS lies within the low normal range [14-15]. Together with the different severities of CFS in the samples tested, the lack of uniformity in exercise testing protocols might account for the differences between results. Both a linear $[4,8-9,13]$ and a stepwise $[10,14]$ increase in bicycle workload have been used to study exercise performance in people with CFS. Therefore, the present study compared the outcome of two exercise testing protocols in patients with CFS: a maximal bicycle exercise test using a linear increase in workload (an increase of $5 \mathrm{~W} / \mathrm{min}$ ) and a maximal bicycle exercise test using a stepwise increase in workload (a sudden increase of $15 \mathrm{~W}$ every $3 \mathrm{~min}$ ). We anticipated that the linear protocol would be more appropriate for women with CFS. The sudden increase of $15 \mathrm{~W}$ might be too difficult for some patients with CFS, possibly causing early cessation of the exercise test and consequent underestimation of peak exercise capacity. We hypothesized that the linear protocol would generate higher peak exercise performance variables compared with the stepwise protocol. We chose the protocols to obtain an equal increase in workload of $15 \mathrm{~W}$ every $3 \mathrm{~min}$ in both protocols (either with a linear or a stepwise increase).

\section{METHODS}

\section{Patient Recruitment and Research Design}

Female patients with CFS were randomly allocated from consecutive referrals to our CFS clinic. Approximately 120 patients were contacted by telephone and asked to participate. Fifty-seven patients agreed to participate. Of these 57 patients, 27 did not attend the scheduled appointments, leaving us with 30 study participants. Data could not be collected during exercise testing for two patients because of technical problems (problems with the printer or the patient being unable to wear the mask throughout the exercise testing session), leaving results from 28 participants. All participants fulfilled the Centers for Disease Control and Prevention (CDCP) criteria for CFS [1]. All patients underwent an extensive medical evaluation prior to study participation (for more details regarding the diagnostic strategies applied in this study, see Nijs et al. [16]). To account for the heterogeneous nature of the CFS population, we applied additional inclusion/exclusion criteria beyond the diagnostic criteria. First, the study focused on women to preclude bias originating from pooling of sex data [14]. Second, all patients spoke Dutch as their native language and were between 18 and 65 years old. The mean age was $42.1 \pm 7.7$ (range $=24-55$ ), and the mean illness duration was $5.5 \pm$ 5.1 years (range $=1-21$ ). Third, study participants had to be able to perform a graded bicycle exercise test. Fourth, all participants had to able to provide written informed consent. An information leaflet was given to all patients, and they were instructed to read it carefully and, if necessary, ask for additional clarification. The study protocol was approved by the local ethics committee (Academical Hospital Vrije Universiteit Brussel, O.G. 016). After providing written informed consent, participants were asked to complete two short questionnaires that would enable us to monitor their health status (the Dutch versions of the CFS Activities and Participation Questionnaire [CFS-APQ] and the CFS Symptom List). Then patients were randomly allocated to either the linear or the stepwise exercise testing protocol (we concealed allocation by having patients draw lots) and performed the exercise test on a bicycle ergometer with continuous monitoring of cardiorespiratory variables. The randomization process did not include any stratification.

\section{Questionnaires}

The CFS-APQ is a self-administered questionnaire that monitors activity limitations and participation restrictions in patients with CFS. A total score of 1 indicates no activity limitations or participation restrictions, while 16 represents the maximum score. Data have been reported that document the test-retest reliability, internal consistency, and content, convergent, and discriminant validity of the Dutch CFSAPQ in patients with CFS [17-18]. The CFS Symptom List is a self-reported measure for assessing symptom severity in patients with CFS. It encompasses the 19 symptoms most frequently reported by a sample of 1,578 patients with CFS [19]. We used visual analog scales $(100 \mathrm{~mm})$ to assess the severity of the symptoms included in the CFS Symptom List. In a previous study of 68 patients with CFS, the internal consistency of the different items included in the Dutch CFS Symptom List was high (Cronbach $\alpha=0.88$ ) [20]. The CFS Symptom List displayed excellent test-retest reliability (intraclass correlation coefficient [ICC] $\geq 0.97$ ), content and concurrent validity.

\footnotetext{
*Nijs J, Thielemans A. Kinesiophobia and symptomatology in chronic fatigue syndrome: a psychometric study of two questionnaires. Unpublished observations; 2007.
} 


\section{Linear Exercise Testing Protocol}

The patients performed a bicycle ergometric test using a linear increase in workload until volitional exhaustion was reached. The patients sat on an electromagnetically braked ergometer (Jaeger 900, Lode B.V., Groningen, the Netherlands). We monitored HR continuously at rest and during exercise using a Polar Accurex Plus $^{\text {TM }}$ (Polar Electro Oy, Kempele, Finland). To collect pulmonary data during the test, we used an open-circuit spirometer (Mijnhart Oxycon, IBM, Bunnik, the Netherlands) with automatic printout every $30 \mathrm{~s}$. Averages were obtained for peak $\mathrm{VO}_{2}$ and maximal carbon dioxide $\left(\mathrm{CO}_{2}\right)$ production during every $30 \mathrm{~s}$ interval for the duration of each stage of the exercise. A two-way breathing valve attached to a mask, which covered the patients' nose and mouth, collected the expired air. The air was analyzed continuously for ventilatory and metabolic variables.

Before each test, we calibrated gas and volume with a 3-l syringe, according to the manufacturer's guidelines. The oxygen $\left(\mathrm{O}_{2}\right)$ analyzer was calibrated with known gas mixtures of 18 percent $\mathrm{O}_{2}$ and 5 percent $\mathrm{CO}_{2}$. The room air calibration was automatically run before each test to update the $\mathrm{CO}_{2}$ analyzer baseline and the $\mathrm{O}_{2}$ analyzer gain so that they coincided with atmospheric values.

Patients started the test at $0 \mathrm{~W}$, with a linear increase of $5 \mathrm{~W} / \mathrm{min}$. Patients were instructed to bicycle at a constant speed of $60 \mathrm{rpm}$. For safety reasons, the investigator ended the test if the patient reached her predetermined agepredicted peak HR or if the cycling speed decreased below $50 \mathrm{rpm}$. The age-predicted peak HR was calculated as 220 minus the patients' age in years. The following variables were measured and computed: HR at rest, peak HR, exercise duration, maximal work capacity attained, work capacity attained at a respiratory exchange ratio (RER) of 1.0 , peak $\mathrm{VO}_{2}$ per kilogram of body weight, body weightadjusted $\mathrm{O}_{2}$ uptake at RER $=1.0$, resting and peak RER, peak ventilation (VE), peak rate of elimination of $\mathrm{CO}_{2}$ $\left(\mathrm{VCO}_{2}\right)$, peak $\mathrm{VE} /$ peak $\mathrm{VCO}_{2}, \mathrm{VO}_{2}$ at $\mathrm{RER}=1.0$ peak $\mathrm{VO}_{2}$, and the percentage of target $\mathrm{HR}$ achieved. The peak workload was divided by peak $\mathrm{VO}_{2}$ to obtain the mechanical efficiency. The metabolic data analyzed were the mean values of the last $30 \mathrm{~s}$ from the final stage of exercise or the highest value attained if a decline in $\mathrm{VO}_{2}$ occurred at the final workload [8]. The study participants were distributed within the four subgroups of the American Medical Association (AMA) impairment rating scale according to the peak $\mathrm{VO}_{2}$ achieved [21]. No impairment was defined as peak $\mathrm{VO}_{2}>25 \mathrm{~mL} / \mathrm{min} / \mathrm{kg}$, mild impairment as peak
$\mathrm{VO}_{2}=20-25 \mathrm{~mL} / \mathrm{min} / \mathrm{kg}$, moderate impairment as peak $\mathrm{VO}_{2}=15-20 \mathrm{~mL} / \mathrm{min} / \mathrm{kg}$, and severe impairment as peak $\mathrm{VO}_{2}<15 \mathrm{~mL} / \mathrm{min} / \mathrm{kg}$ [21].

\section{Stepwise Exercise Testing Protocol}

The patients performed a bicycle ergometric test using a stepwise increase in workload until volitional exhaustion was reached. All patients started the test at $15 \mathrm{~W}$, with a sudden increase of $15 \mathrm{~W}$ every $3 \mathrm{~min}$. Apart from the way the workload was increased, the methods described for the linear exercise testing protocol apply to the stepwise exercise testing protocol.

\section{Statistical Analysis}

We analyzed all data using SPSS $11.0^{\circledR}$ for Windows (SPSS Inc, Chicago, Illinois). We used a one-sample Kolmogorov-Smirnov (K-S) goodness-of-fit test to examine whether the variables were normally distributed. In cases of normally distributed variables, we used the independent samples Student's $t$-test to compare the two groups. If the K-S revealed that a variable was not normally distributed, then the nonparametric Mann-Whitney test was used. In addition to the ratio peak workload/peak $\mathrm{VO}_{2}$ as a measure of mechanical efficiency, the linear regression slope values between $\mathrm{VO}_{2}$ (dependent variable) and workload (independent variable) from the minute-by-minute exercise data points for each subject were computed, and the mean values were compared between groups with the Student's $t$-test. When appropriate, the significance level was set at 0.01 to help protect against potential type I errors.

\section{RESULTS}

Apart from illness duration (K-S z-score $=1.4$; $p=$ $0.04)$, all health status variables and demographic features were normally distributed. Both groups $(n=14)$ were similar in demographic features (age, illness duration, height, and weight) and health status (symptom severity and activity limitations/participation restrictions) (Table 1). Besides the lack of difference in total score on the CFS Symptom List (displayed in Table 1), no differences in any of the 19 individual visual analog symptom scales were found (data not shown).

Two exercise tests were terminated because the subject reached the predetermined age-predicted peak HR. The distribution of the sample within the four subgroups 
of the AMA impairment rating scale [21] is presented in Table 2; the majority of the CFS patients $(18 / 28$ or $64 \%$ ) are considered to have "moderate to severe impairment of the whole person" (classes 3 and 4). The distribution of the participants within the four subgroups was identical for both groups (stepwise vs linear exercise protocol) (Table 2). Apart from the workload at $\mathrm{RER}=1.0(\mathrm{~K}-\mathrm{S} z$-score $=1.5 ; p=0.01)$, all exercise performance variables were normally distributed. The descriptives and comparative statistics of the exercise performance data are presented in Table 3. Based on the ratio peak workload/peak $\mathrm{VO}_{2}$, mechanical efficiency was lower among the subjects performing the stepwise protocol $(p=0.002)$. When analyzing mechanical efficiency using the mean linear regression slope values between $\mathrm{VO}_{2}$ and workload from the minute-by-minute exercise data points for each subject, we observed a group difference. Compared with the group performing the linear protocol $(7.87 \pm 3.29)$, the mean \pm standard deviation slope value was higher in the group performing the graded exercise protocol $(10.58 \pm 3.12)(t=-2.18$; $p=0.039$ ). Apart from the mechanical efficiency, we found no differences in exercise performance data between groups. Based on peak VE/peak $\mathrm{VCO}_{2}$, ventilatory drive was similar in both groups.

\section{DISCUSSION}

The results suggest that the nature of the exercise testing protocol influences the mechanical efficiency during exercise of women with CFS. Compared with the subjects performing the exercise test with a linear increase in workload (5 W/min), mechanical efficiency was lower among the subjects performing the exercise test with a sudden increase of $15 \mathrm{~W}$ every $3 \mathrm{~min}$ (stepwise protocol). Mechanical efficiency was lower both at the termination of the exercise test (i.e., the ratio peak workload/peak $\mathrm{VO}_{2}$ ) and during exercise (i.e., the slope values). Still, the results suggest that the difference between the linear and stepwise exercise protocols cannot account for all discrepancies of previous studies on exercise performance in women with CFS. Indeed, the majority of the submaximal and peak exercise performance data (e.g., $\mathrm{VO}_{2}$ at RER = 1.0, peak $\mathrm{VO}_{2}$, peak VE peak RER, peak HR) did not differ between groups. Apart from the mechanical efficiency, the results suggest that a maximal bicycle exercise test using a linear increase in workload generates results similar to a maximal bicycle exercise test using a stepwise increase in workload. Contrary to what we anticipated before the study, the results do not suggest that the sudden

Table 1.

Comparison of demographic features and health status between linear $(n=14)$ and stepwise $(n=14)$ exercise testing protocol groups.

\begin{tabular}{lccc}
\hline \multicolumn{1}{c}{ Variable } & $\begin{array}{c}\text { Linear Protocol } \\
\text { Mean } \pm \text { SD (Range) }\end{array}$ & $\begin{array}{c}\text { Stepwise Protocol } \\
\text { Mean } \pm \text { SD (Range) }\end{array}$ & p-Value \\
\hline Age (yr) & $40.3 \pm 8.8(24-52)$ & $43.9 \pm 6.2(31-55)$ & 0.22 \\
Height (cm) & $164.7 \pm 4.7(157-173)$ & $161.7 \pm 5.9(152-173)$ & 0.15 \\
Weight (kg) & $66.4 \pm 9.9(51-79)$ & $62.2 \pm 11.0(49-81)$ & 0.30 \\
Illness Duration (yr) & $5.2 \pm 4.6(1-17)$ & $5.9 \pm 5.9(1-21)$ & $0.97^{*}$ \\
CFS-APQ & $7.2 \pm 2.1(4.2-10.2)$ & $8.4 \pm 2.8(3.9-13.9)$ & 0.22 \\
CFS Symptom List & $43.7 \pm 20.7(10.0-75.7)$ & $43.0 \pm 15.4(12.8-66.3)$ & 0.92 \\
\hline
\end{tabular}

${ }^{*}$ Nonparametric Mann-Whitney test.

CFS = chronic fatigue syndrome, CFS-APQ = CFS Activities and Participation Questionnaire, SD = standard deviation.

Table 2.

Distribution of sample $(n=28)$ and both exercise protocol groups within four subgroups of American Medical Association (AMA) impairment rating scale*.

\begin{tabular}{lllll}
\hline \multicolumn{1}{c}{ Subjects } & Class 1 & Class 2 & Class 3 & \multicolumn{1}{c}{ Class 4 } \\
\hline All Subjects, $n(\%)$ & $2(7.1)$ & $8(28.6)$ & $8(28.6)$ & $5(35.7)$ \\
Linear Group, $n$ & 1 & 4 & 4 & 5 \\
Stepwise Group, $n$ & 1 & 4 & 4 & 5
\end{tabular}

*AMA impairment rating scale definitions—Class 1: 0\% degree of impairment (no impairment), maximal $\mathrm{O}_{2}$ consumption >25 mL/min/kg; Class 2: 10\%-25\% degree of impairment (mild impairment), maximal $\mathrm{O}_{2}$ consumption $20-25 \mathrm{~mL} / \mathrm{min} / \mathrm{kg}$; Class 3: 30\%-45\% degree of impairment (moderate impairment), maximal $\mathrm{O}_{2}$ consumption 15-20 mL/min/kg; and Class 4: 50\%-100\% degree of impairment (severe impairment), maximal $\mathrm{O}_{2}$ consumption $<15 \mathrm{~mL} / \mathrm{min} / \mathrm{kg}$. 
Table 3.

Comparison of exercise performance data between linear $(n=14)$ and stepwise $(n=14)$ exercise testing protocol groups.

\begin{tabular}{|c|c|c|c|}
\hline Variable & $\begin{array}{c}\text { Linear Protocol } \\
\text { Mean } \pm \text { SD (range) }\end{array}$ & $\begin{array}{c}\text { Stepwise Protocol } \\
\text { Mean } \pm \text { SD (range) }\end{array}$ & $p$-Value \\
\hline Peak HR (bpm) & $150.4 \pm 22.6(104-174)$ & $140.3 \pm 26.6(104-185)$ & 0.29 \\
\hline \% Age-Predicted Peak HR & $83.6 \pm 11.5$ (57.4-95.9) & $79.7 \pm 14.8$ (60.5-101.6) & 0.44 \\
\hline Peak Workload (W) & $97.1 \pm 30.4(40-170)$ & $77.9 \pm 42.7(30-190)$ & 0.18 \\
\hline Peak $\mathrm{VO}_{2}(\mathrm{~mL} / \mathrm{min})$ & $1,124.4 \pm 408.2(317-1,697)$ & $1,150.9 \pm 462.7(670-2,518)$ & 0.87 \\
\hline Peak $\mathrm{VO}_{2}(\mathrm{~mL} / \mathrm{min} / \mathrm{kg})$ & $17.2 \pm 6.4(5.6-25.4)$ & $18.7 \pm 6.5(8.8-33.6)$ & 0.43 \\
\hline Peak $\mathrm{VCO}_{2}(\mathrm{~L} / \mathrm{min})$ & $1.24 \pm 0.51(0.23-2.06)$ & $1.23 \pm 0.53(0.66-2.60)$ & 0.99 \\
\hline Peak VE (L/min) & $44.34 \pm 18.60(8.70-70.57)$ & $44.76 \pm 20.65(22.20-104.30)$ & 0.96 \\
\hline Peak VE/Peak $\mathrm{VCO}_{2}$ & $36.6 \pm 7.8(23-57)$ & $36.7 \pm 6.8(26-47)$ & 0.97 \\
\hline Peak Workload/Peak VO $2\left(\mathrm{~W} \cdot \mathrm{mL}^{-1} \cdot \mathrm{min}^{-1} \cdot \mathrm{kg}^{-1}\right)$ & $6.24 \pm 2.22(3.41-12.07)$ & $3.95 \pm 0.92(2.13-5.66)$ & 0.002 \\
\hline $\mathrm{HR}$ at $\mathrm{RER}=1.0(\mathrm{~W})$ & $124.5 \pm 15.4(110-162)$ & $119.2 \pm 21.7(88-163)$ & 0.51 \\
\hline
\end{tabular}

increase of $15 \mathrm{~W}$ (stepwise protocol) causes early cessation of the exercise test and consequent underestimation of peak exercise capacity in women with CFS. Apart from the mechanical efficiency, the results refute our hypothesis that the linear protocol generates higher submaximal or peak exercise performance variables compared with the stepwise protocol. The observation that the subjects had lower mechanical efficiency performing the stepwise protocol may be valuable to healthcare workers and researchers applying exercise testing and exercise therapy in women with CFS.

We chose the protocols to obtain an equal increase in workload of $15 \mathrm{~W}$ every $3 \mathrm{~min}$ in both protocols. Further study is warranted, for instance, on other exercise testing protocols previously used for studying patients with CFS. Fulcher and White used a treadmill walking test at a constant speed of $5 \mathrm{kph}$ and a gradient increase of 2.5 percent every 2 min [11]. Sargent et al. applied cycle ergometry starting at $0 \mathrm{~W}$ by incrementing the power output by $25 \mathrm{~W}$ every 2 min [14]. Bazelmans and colleagues applied an individually tailored bicycle ergometer test: the workload was increased every minute in steps of 10 percent of estimated maximal workload [4]. Future studies are required to reveal whether these protocols generate similar exercise performance data in patients with CFS and to identify the optimal exercise testing protocol for individuals with CFS. In addition, a future study should compare the mechanical efficiency of people with CFS and nondisabled sedentary controls.

Apart from the lack of uniformity in exercise testing protocols, the highly heterogeneous nature of the CFS population and the lack of uniformity in the diagnostic criteria used may account for the differences between investigators. Indeed, a previous study showed that a method for predicting peak $\mathrm{VO}_{2}$ established in Oxford-defined patients with CFS was not applicable to CDCP-defined patients with CFS [22]. Compared with the 1994 CDCP case definition for CFS [1], the British criteria for CFS [23] do not include symptoms of depressive illness and anxiety disorders as exclusion factors and use fewer symptom criteria.

Our performance data, interpreted according to the AMA impairment rating scale, support previous studies suggesting a reduced exercise capacity in patients with CFS [8,10-13]. In analysis of the mean peak oxygen data of both groups, the women with CFS remained 27 to 32 percent below the threshold value for having "no impairment of the whole person" defined by the scale of impairment rating [21]. Recent studies provided insight into the nature of abnormal exercise performance in people with CFS: kinesiophobia (irrational fear of movement) was not related to exercise performance [24], but intracellular immune deregulations were identified as determinants of exercise performance [16,25]. In addition, patients with CFS have been shown to respond to an exercise challenge 
with a lengthened and accentuated oxidative stress response [24], an enhanced complement activation [26], and exaggerated resting differences in gene expression profile in peripheral blood mononuclear cells [27].

Our results should be interpreted in light of the study limitations. First, having all patients perform both exercise testing protocols would have been the primary choice for our study design. We anticipated that patients with CFS would be unable to perform more than one maximal exercise test a day. Indeed, experimental data shows that the majority of patients with CFS relapse following maximal exertion [5]. Therefore, asking the patients to return for a second maximal exercise test days or even weeks after the first assessment was deemed medically risky and therefore unethical. In addition, having the study participants return for the second test performance might have introduced potential bias from the fluctuating nature of CFS symptoms. Given the lack of differences in symptom severity and activity limitations/participation restrictions between groups, the results presented here are unlikely to be biased by differences in health status. For all these reasons, the study design was deemed appropriate for examining the study aim. Second, the results are applicable to women attending a specialized CFS clinic. Male or primary care CFS patients were not studied. Third, the patients studied here were distributed within the four subgroups of the AMA scale of impairment rating, suggesting a heterogeneous group of patients. While heterogeneity in exercise testing protocols might be an issue in CFS research, the heterogeneous nature of the CFS population is likely to be another contributor to conflicting research results among researchers.

\section{CONCLUSIONS}

In light of the study limitations, our results suggest that the nature of the exercise testing protocol influences mechanical efficiency during exercise in women with CFS. Compared with the subjects performing the exercise test with a linear increase in workload, mechanical efficiency was lower among the subjects performing the exercise test with a stepwise protocol. In addition, our study of women with CFS suggests that a maximal bicycle test using a linear increase in workload generates similar $\mathrm{VO}_{2}$ at $\mathrm{RER}=1.0$, peak $\mathrm{VO}_{2}$, peak VE, peak RER, peak HR results as a maximal bicycle test using a stepwise increase in workload. The sudden increase of
$15 \mathrm{~W}$ (stepwise protocol) did not significantly reduce the peak $\mathrm{VO}_{2}$ estimate. According to these findings, the difference between linear and stepwise exercise protocols cannot account for all discrepancies of previous studies on exercise testing in CFS.

\section{ACKNOWLEDGMENTS}

This work was unfunded at the time of manuscript preparation.

The authors have declared that no competing interests exist.

\section{REFERENCES}

1. Fukuda K, Strauss SE, Hickie I, Sharpe MC, Dobbins JG, Komaroff A. The chronic fatigue syndrome: A comprehensive approach to its definition and study. International Chronic Fatigue Syndrome Study Group. Ann Intern Med. 1994;121(12):953-59. [PMID: 7978722]

2. Clapp LL, Richardson MT, Smith JF, Wang M, Clapp AJ, Pieroni RE. Acute effects of thirty minutes of light-intensity, intermittent exercise on patients with chronic fatigue syndrome. Phys Ther. 1999;79(8):749-56. [PMID: 10440661$]$

3. Black CD, O'Connor PJ, McCully KK. Increased daily physical activity and fatigue symptoms in chronic fatigue syndrome. Dyn Med. 2005;4(1):3. [PMID: 15745455]

4. Bazelmans E, Bleijenberg G, Voeten MJ, Van der Meer $\mathrm{JW}$, Folgering $\mathrm{H}$. Impact of a maximal exercise test on symptoms and activity in chronic fatigue syndrome. J Psychosom Res. 2005;59(4):201-8. [PMID: 16223622]

5. Lapp CW. Exercise limits in chronic fatigue syndrome. Am J Med. 1997;103(1):83-84. [PMID: 9236491]

6. Paul L, Wood L, Behan WM, Maclaren WM. Demonstration of delayed recovery from fatiguing exercise in chronic fatigue syndrome. Eur J Neurol. 1999;6(1):63-69. [PMID: 10209352]

7. Manu P, Affleck G, Tennen H, Morse PA, Escobar JI. Hypochondriasis influences quality-of-life outcomes in patients with chronic fatigue. Psychother Psychosom. 1996;65(2): 76-81. [PMID: 8711085]

8. De Becker P, Roeykens J, Reynders M, McGregor N, De Meirleir K. Exercise capacity in chronic fatigue syndrome. Arch Intern Med. 2000;160(21):3270-77. [PMID: 11088089]

9. Nijs J, De Meirleir K, Wolfs S, Duquet W. Disability evaluation in chronic fatigue syndrome: Associations between exercise capacity and activity limitations/participation restrictions. Clin Rehabil. 2004;18(2):139-48. [PMID: 15053122] 
10. Wallman KE, Morton AR, Goodman C, Grove R. Physiological responses during a submaximal cycle test in chronic fatigue syndrome. Med Sci Sports Exerc. 2004;36(10): 1682-88. [PMID: 15595287]

11. Fulcher KY, White PD. Strength and physiological response to exercise in patients with chronic fatigue syndrome. J Neurol Neurosurg Neuropsychiatry. 2000;69(3): 302-7. [PMID: 10945803]

12. Paul LM, Wood L, Maclaren W. The effect of exercise on gait and balance in patients with chronic fatigue syndrome. Gait Posture. 2001;14(1):19-27. [PMID: 11378421]

13. Wong R, Lopaschuk G, Zhu G, Walker D, Catellier D, Burton D, Teo K, Collins-Nakai R, Montague T. Skeletal muscle metabolism in the chronic fatigue syndrome: In vivo assessment by 31P nuclear magnetic resonance spectroscopy. Chest. 1992;102(6):1716-22. [PMID: 1446478]

14. Sargent C, Scroop GC, Nemeth PM, Burnet RB, Buckley JD. Maximal oxygen uptake and lactate metabolism are normal in chronic fatigue syndrome. Med Sci Sports Exerc. 2002;34(1):51-56. [PMID: 11782647]

15. Gallagher AM, Coldrick AR, Hedge B, Weir WR, White $\mathrm{PD}$. Is the chronic fatigue syndrome an exercise phobia? A case control study. J Psychosom Res. 2005;58(4):367-73. [PMID: 15992572]

16. Nijs J, Meeus M, McGregor NR, Meeusen R, De Schutter G, Van Hoof E, De Meirleir K. Chronic fatigue syndrome: Exercise performance related to immune dysfunction. Med Sci Sports Exerc. 2005;37(10):1647-54. [PMID: 16260962]

17. Nijs J, Vaes P, McGregor N, Van Hoof E, De Meirleir K. Psychometric properties of the Dutch Chronic Fatigue Syndrome-Activities and Participation Questionnaire (CFSAPQ). Phys Ther. 2003;83(5):444-54. [PMID: 12718710]

18. Nijs J, Cloostermans B, McGregor N, Vaes P, De Meirleir K. Construct validity and internal consistency of the chronic fatigue syndrome activities and participation questionnaire (CFS-APQ). Physiother Theory Pract. 2004;20(1):31-40.

19. De Becker P, McGregor N, De Meirleir K. A definitionbased analysis of symptoms in a large cohort of patients with chronic fatigue syndrome. J Intern Med. 2001;250(3): 234-40. [PMID: 11555128]
20. Nijs J, Aerts A, De Meirleir K. Generalized joint hypermobility is more common in chronic fatigue syndrome than in healthy control subjects. J Manipulative Physiol Ther. 2006; 29(1):32-39. [PMID: 16396727]

21. Make B, Jones JF. Impairment of patients with chronic fatigue syndrome. J Chronic Fatigue Syndr. 1997;3(4):43-56.

22. Nijs J, De Meirleir K. Prediction of peak oxygen uptake in patients fulfilling the 1994 CDC criteria for chronic fatigue syndrome. Clin Rehabil. 2004;18(7):785-92.

[PMID: 15573835]

23. Sharpe MC, Archard LC, Banatvala JE, Borysiewicz LK, Clare AW, David A, Edwards RH, Hawton KE, Lambert HP, Lane RJ. A report-Chronic fatigue syndrome: Guidelines for research. J R Soc Med. 1991;84(2):118-21. [PMID: 1999813]

24. Nijs J, De Meirleir K, Duquet W. Kinesiophobia in chronic fatigue syndrome: Assessment and associations with disability. Arch Phys Med Rehabil. 2004;85(10):1586-92. [PMID: 15468015]

25. Snell CR, Vanness JM, Strayer DR, Stevens SR. Physical performance and prediction of 2-5A Synthetase/RNase L antiviral pathway activity in patients with chronic fatigue syndrome. In Vivo. 2002;16(2):107-9. [PMID: 12073768]

26. Jammes Y, Steinberg JG, Mambrini O, Brégeon F, Delliaux S. Chronic fatigue syndrome: Assessment of increased oxidative stress and altered muscle excitability in response to incremental exercise. J Intern Med. 2005; 257(3):299-310. [PMID: 15715687]

27. Sorensen B, Streib JE, Strand M, Make B, Giclas PC, Fleshner M, Jones JF. Complement activation in a model of chronic fatigue syndrome. J Allergy Clin Immunol. 2003; 112(3):397-403. [PMID: 12897748]

28. Whistler T, Jones JF, Unger ER, Vernon SD. Exercise responsive genes measured in peripheral blood of women with chronic fatigue syndrome and matched control subjects. BMC Physiol. 2005;5(1):5. [PMID: 15790422]

Submitted for publication December 6, 2006. Accepted in revised form February 21, 2007. 